\title{
MARPLE Investigates: An 'Adversarial' Approach to Evaluating User Experience
}

\author{
Jane Holt \\ Lancaster University \\ Computing Department \\ Infolab 21 \\ +44 (0) 1524510311 \\ holtj1@comp.lancs.ac.uk
}

\author{
Simon Lock \\ Lancaster University \\ Computing Department \\ Infolab 21 \\ +44 (0) 1524510304 \\ lock@comp.lancs.ac.uk
}

\begin{abstract}
User experience of interactive systems has always been difficult to assess due to its subjective nature. In this paper we present a new approach to the evaluation of pleasure as an aspect of user experience. This multi-lateral approach, entitled MARPLE, is based upon an adversarial courtroom metaphor.
\end{abstract}

\section{Categories and Subject Descriptors}

H1.2 User/Machine Systems; H5. Information Interfaces and Presentation.

\section{Keywords}

User Experience, Interactive, Subjective, Evaluation, Adversarial.

\section{INTRODUCTION}

The last ten years has seen a growth of interest, within the Human-Computer Interaction (HCI) community, towards User Experience. User experience is concerned with the 'quality of the process' that the user feels during interaction. User experience typically includes such aspects as fun [4, 22], creativity [3, 15], playfulness [14] and aesthetics [15]. Efforts are currently being made to design and evaluate systems which take these aspects into account. However, this is a challenging prospect due to the emotional aspects of user experience.

The term 'emotion' is in itself difficult to define. There are many differing opinions as what emotion is and how it functions, from such fields as psychology [6, 7, 9, and 11] and neuroscience [19]. What appears to be a repeating theme though, is that emotion is seen as 'a mental state that does not arise through free will, and that is often accompanied by physiological changes' [9]. These states can be triggered by either an internal or external stimulus, e.g. a pleasant memory or a perceived threat, which can then manifest themselves as an increase in heart rate and breathing, changes in facial expression or tensing of the muscles. There are said to be six basic emotions that we all experience, such as anger, disgust, fear, joy, sadness and surprise [6], though the number and type varies amongst psychologists [6, 7, 9 and 11].

(C) Jane Holt and Simon Lock 2007.

Published by the British Computer Society

Volume 2 Proceedings of the $21^{\text {st }}$ BCS HCI Group Conference

HCI 2007, Lancaster University, 3 - 7 September 2007
Emotional states are both fleeting and unpredictable, and are open to individual interpretation which can also be subjective. What is interesting to note is that historically, emotions were seen in a negative aspect, as self-limiting and obstructive as compared to a logical, rational approach. However, due to research undertaken into the field of affective computing, emotions have come to the forefront with the emphasis now on ensuring systems are enjoyable as well as usable.

Incorporating this into the design process is problematic, and this equally applies to evaluation. Usability has long established

methods in order to determine whether a system is efficient, effective and satisfying. Conversely, user experience is a relatively new area which is focused upon developing frameworks and approaches [3, 8, 12], which will include evaluation guidance. Experiments have been conducted into this type of evaluation [1,] which draw upon existing usability techniques, such as questionnaires [5, 18], interviews, [5, 18], heuristics [18] and 'think aloud' protocols [17]. These are well used techniques, in both academia and industry, which appear to provide reliable data.

However, user experience is a wide ranging area of research which has engendered a variety of approaches. Many researchers are adopting a 'holistic' approach by focusing on the subject as a whole $[3,8]$, whereas others have chosen to concentrate on single aspects such as fun [22, 4], enjoyment [17], play [15] and motivation [8].

\section{EXISTING APPROACHES}

Currently there appears to be no single standard approach to the issue of evaluating pleasure, as part of user experience. Discussion is centred around whether to rework 'old' or existing methods of evaluation, specifically for user experience, or to develop new techniques. Another option is that of combining techniques from usability with user experience thereby assessing both task performance and emotional impact.

What is recognized by many researchers is the challenge of designing a common methodological approach [3] for this purpose. It has even been suggested that this may be an insurmountable problem due to the imprecise and ambiguous nature of emotions. However, we have already mentioned that there is a growing awareness of the importance of emotions during user interaction and so efforts are being made to find ways of assessing their impact.

We have already mentioned that some researchers have chosen to use existing usability techniques, such as interviews - both structured and semi structured [5, 18] and questionnaires [5, 18]. These and other usability methods are frequently used throughout the usability community and as such can be said to 
be tried and tested though not entirely conclusive. This may be more of an issue with qualitative methods which tend to rely upon interpretation of data rather than pure statistics [18].

As well as these, other approaches are being used: one approach uses bio sensing or physiological measures. These are metrics which allow us to obtain physiological data in order to assess user emotion and stress [14]. Sometimes used in cognitive psychology, this includes measuring heart rate via an Electrocardiogram (ECG) or the conductivity of the skin via Galvanic Skin Response (GSR). Increases in heart rate or the temperature of the skin can indicate periods of stress and anxiety experienced by the user during interaction.

Another is that of 'pastiche scenarios', which a team at the University of York have been using as a form of evaluation. These are short narratives which borrow from other texts to show how a user might interact with a device. Similar to a diary yet also closely allied to persona creation, they aim to capture emotionally rich data from everyday occurrences [2].

A more structured method is advocated, based upon an experiential value scale which measures user responses via a seven point scale. Based upon marketing research, it uses nine indicators, for example, intrinsic enjoyment, that are then used against a series of statements related to user experience. These statements are measured via this scale according to their levels of agreements [21].

Other new methods have been proposed, for example, 'cultural probes' in which users self report about their interaction experience via a package consisting of a diary, camera and task cards, which records their actions, thoughts and feelings [1, 10]. The use of 'anticipation' interviews in which users are encouraged to keep voice note diaries, followed by a reflection interview [20], and a multiple method which uses in situ observations, 'obstacle cards' and interviews, at various stages in order to assess customer behaviour for an e-commerce website [16].

We have highlighted a few of the various approaches, for user experience evaluation, which are drawn from both the qualitative and quantitative domains. What is interesting to note is that some of these use a single technique for evaluation whereas others suggest a multiple approach. Which are better for user experience evaluation? Both of these approaches have advantages and disadvantages which are discussed in the next section.

\section{COMPARISON AND CRITIQUE}

The use of a single technique, for example, a questionnaire, may be considered perfectly appropriate for user experience evaluation. These have been and are used on a regular basis in both academia and industry: a single technique could be particularly beneficial when both time and money are subject to constraints, for example, conducting an 'expert walkthrough' [18]. On the other hand, if undertaking a protracted series of evaluations, then the utilisation of several techniques may be more profitable. In either case, a systematic approach seems to bring dividends - when determining usability. Usability is often focussed upon the achievement of specific criteria [18] within a context of use, so having what appear to be clearly defined goals and objectives can make things easier.

However, the application of a rigid 'checklist' approach to what can be a fluid, unpredictable and sensual process may not be ideal. It may be more beneficial to use a range of techniques which are based upon interpretation rather than metrics, for example, combining interviews with video observation and/or photography, or cultural probes followed by an interview. As we are dealing with qualitative measures, a single technique might not be strong enough or reliable enough to accurately capture data. So it may be more worthwhile to use several of these techniques together in order to provide a solid justification when discussing the results.

\section{THE MARPLE METHOD}

MARPLE stands for the Multi-lateral Assessment and Review of Pleasure Laden Experiences and is a methodology which is currently being developed as a response to the issue of evaluating pleasure as part of the user experience. Due to its interpretative nature, we are devising a largely qualitative approach which is both systematic and integrated. It is still at an early stage, though it will undergo further refinement over time via testing and feedback. It will use several existing techniques e.g. interviews, video observation etc, as a means of gathering evidence, which is then used in a post-session discussion. As illustrated in figure 1, MARPLE will aim to provide a framework which drives these individual approaches, integrates their results and supports the building of cases to argue about specific aspects of user.

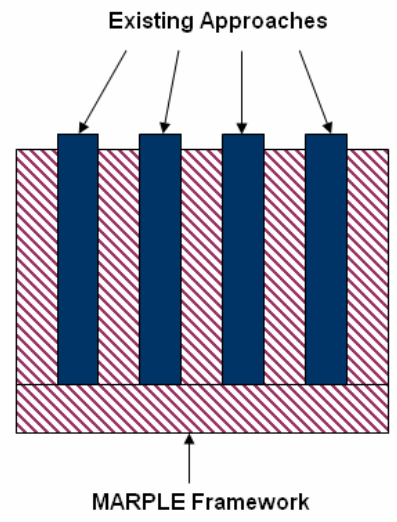

Figure 1. The MARPLE integrated approach

In keeping with the name and the 'adversarial' metaphor, this methodology aims to build a case for the assessment of user experience, from which it will be decided if it was pleasurable or whether in fact, it was 'displeasurable'. It will use these techniques, in conjunction with each other, to gather evidence, which is then offered as proof at the post session discussion. A single piece of evidence could be seen as too flimsy and not capable of fully capturing the emotional aspects whereas several pieces of evidence, used together could help reinforce an argument.

We also advocate the use of two investigators who will take up positive and negative stances, or in reference to the adversarial metaphor, the 'prosecution' and the 'defence'. These investigators will be looking to determine if the interaction was pleasurable or not and the reasons why. The rationale behind this stance is that they may be able to counter accusations of personal subjectivity and bias. One will argue for the positive determinants of a pleasurable interaction and the other will argue for the negative aspects. Then, the two will swap roles and continue the discussion until a final judgement is reached via a third party. The MARPLE Method is strongly based upon a prosecuting/defence investigator stance which we think 
makes it a novel approach to the issue of user experience evaluation.

MARPLE will consist of two halves: one half is the theoretical background and the other is the development of a 'toolkit' which can be used to conduct evaluation of interaction experiences. This toolkit will include a set of guidelines as to what to consider during evaluation, a tutorial which outlines the pleasure evaluation process, from the initial setting up of the equipment through to data analysis and post session discussion, and a case study. Its systematic approach includes obtaining participants, the setting up of the test situation, the evaluation session using video and interviews, data analysis and then the post session discussion with the prosecuting/defence stance. The post session discussion will be followed by the production of an evaluation report.

The MARPLE process model provides an overall framework for structured evidence gathering, analysis and interpretation. This model starts with the setting up of the system, the interaction itself and evidence gathering, through to data analysis, the post session discussion and the creation of a case for a positive or negative experience.

MARPLE will be used at various stages throughout the design cycle, where evaluation needs to be undertaken, e.g. low fidelity stage, and, after implementation, before the system is released into the public domain. It is also designed to complement current usability methods.

\section{EVALUATION: HCI 2006}

MARPLE was first trialled at the Re (Actor) workshop, as part of HCI 2006, in which it assessed the pleasurability of an interactive installation. In order to do this, we set up a 3D interactive installation (illustrated in figure 2) which used two anthropomorphic interface agents, a games console and two unusual input devices. The two unusual input devices - a couple of children's dolls were chosen as firstly, the theme of the workshop was 'playful/clubbing environments'; secondly, we thought they epitomised 'play' as well as being amusing and thirdly, that they would engender a pleasurable experience.

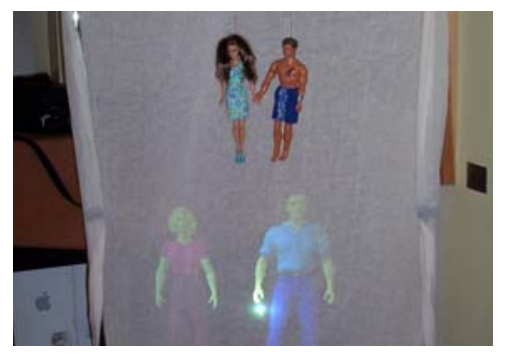

Figure 2. Interactive Installation (HCI 2006)

We set up the interactive installation in an area in which other types of 'playful' devices were displayed. During the conference intervals, e.g. lunch, delegates could wander around and try out the devices. The installation was based upon users being able to emotionally interact with the two agents, by manipulating the two dolls. He/she could express a single emotion which the agents would respond to from a pre-defined range of actions. For example, if the user wished to show 'anger', he/she would shake the dolls violently or use them to perform 'punching' actions towards the agents who would respond by 'punching' back towards the user. The agents always had a default 'start' position in which they were standing still facing the user. The installation involved the user performing a single action with the dolls to which the agents would respond accordingly and then return to the start position. The user would perform another action; the agents would respond again, assume the start position and so on. The agents, one male and one female, were constructed using Poser 4 - 3D character modelling software, and the interactivity was enabled using Java and the console's basic motion capture system.

Due to time constraints, we were able to perform 'quick and dirty' evaluations with five users only. These users were mainly from the performing arts/digital arts domains and were evenly balanced in terms of gender, age and expertise. Each user would sit in front of the installation and would emotionally interact with two 3D interface agents via the doll input devices. These dolls were attached via retractable wires to a games console which the user could manipulate in any way they saw fit - see Figure 2. Each session involved an informal interview with video observation and photography and lasted, on average, around five to ten minutes.

This was followed by data analysis of the interview transcripts and a post session discussion. The data analysis involved grouping the stills into a linear sequence or 'storyboards', to ascertain a pattern of interaction and any distinguishing features. The video footage was viewed in much the same way, with the emphasis on looking at facial expression, gesture and body language in order to highlight positive/negative aspects.

Similarly with the interview transcripts: these were analysed in order to look for repeating words or emerging patterns of behaviour. As the interviews themselves were short, this meant a limited amount of user feedback, though we still managed to elicit some useful comments. The results showed that overall, the users enjoyed the interactive installation, with such words as 'playful', 'funny' and 'good' which suggest that this was a positive experience. There were a few suggestions for improving the installation, such as the 'time lag' between the user's action and the agent's reaction.

The resulting data was then open to discussion by both investigators who adopted the prosecuting and defence lawyer stance. One investigator argued in favour of a positive experience by describing various features which they felt supported this; such as expressive body language, smiling face etc, whereas the other investigator opposed this by pointing out indicators of a negative experience. Eventually, agreement was reached on a variety of issues though it was suggested that a neutral party might be needed.

This initial trial was fairly successful in that it elicited positive and useful feedback. It also raised a number of important issues relating to the evaluation of user experience in general. One such issue is the problem of interpreting photographic evidence, and as such, can not be taken as conclusive. Also, one aspect of MARPLE still open to debate is the friendly, 'chatty' and yet semi structured interview or 'stealth interviewing' which is designed to be unobtrusive and still obtain useful feedback. We have designed a series of suggested interview questions, which are concerned with conceptual issues rather than issues of 'how do you do this', 'what did you expect to happen' and so on. There is the tendency to slip into usability interviewing, particularly if the investigator has previous experience in this area and so there needs to be an awareness of this issue. 
The MARPLE Method is still in its infancy and so further work needs to be undertaken before advocating its adoption by both academia and industry. This means a look at other multifaceted approaches, an appraisal of the techniques we have chosen and why, and, its suitability and appropriateness as a means of evaluating user experience.

\section{REFERENCES}

[1] Axelrod, L and Hone, K.S. Affectemes and Allafects: a novel approach to coding user emotional expression during interactive experiences. Behaviour and Information Technology, Vol. 25, No 2, March-April 2006, pp 159-173.

[2] Blythe, M. Pastiche Scenarios. Interactions, September + October 2004.

[3] Blythe, M; Wright, P; McCarthy, J and Bertelsen, O.W. Theory and Method for Experience Centred Design. CHI 2006, April 22-27, 2006, Montreal, Quebec, Canada.

[4] Carroll, J.M. Beyond Fun. Interactions, September 2004.

[5] Dix, A; Finlay, J; Abowd, G.D; and Beale, R. Human-Computer Interaction: Third Edition. Pearson Prentice Hall, Essex, England, 2004.

[6] Ekman, P. Facial Expression and Emotion. 1992 Award Address. American Psychologist, April 1993, Vol 48, No 4, pp 384-392.

[7] Ekman, P. Basic Emotions. Chapter 3, Handbook of Cognition and Emotion. Sussex, UK. John Wiley \& Sons Ltd, 1999.

[8] Forlizzi, J and Battarbee, K. Understanding Experience in Interactive Systems. DIS2004, August 1-4, 2004, Cambridge, Massachusetts.

[9] Goertzel, B. A General Theory of Emotion in Humans and Other Intelligences. DynaPsych Table of Contents. http://www.goertzel.org/dynapsyc/2004/Emotions.htm.

[10] Jaasko, V and Mattelmaki, T. Observing and Probing. DPPI '03, June 23-26, 2003, Pittsburgh, Pennsylvania, USA.

[11] James, W. What is an Emotion? Classics in the History of Psychology. An internet resource developed by Christopher D. Green, York University, Toronto, Ontario. http://psychclassics.yorku.ca/James/emotion.htm.

[12] Knight, J and Jefsioutine, M. The Experience Design Framework: from Pleasure to Engagability. Conference HCI, the Arts and Humanities, 2003, University of York,
UK. http://www-users.cs.york.ac./

$\sim p c w / K M \_s u b s /$ Knight_Jefsioutine.pdf.

[13] Mandryk, R.L; Atkins, M.S and Inkpen, K.M.

A Continuous Objective Evaluation of Emotional Experience with Interactive Play Environments. Proceedings of the SIGCHI conference on Human Factors in computing systems, Novel Methods: Emotions, Gestures, Events (CHI 2006), (Montreal, Quebec, April 22-27, 2006).

[14] Mandryk, R.L; Inkpen, K.M and Calvert, T.W. Using psychophysiological techniques to measure user experience with entertainment technologies. Behaviour and Information Technology, Vol 25, No. 2, March-April 2006, pp 141-158.

[15] Myers, D. What's good about bad play? Proceedings of the Second Australasian Conference on Interactive Entertainment, 2005, Sydney, Australia 2004.

[16] Petre, M; Minocha, S; and Roberts, D. Usability beyond the website: an empirically-grounded e-commerce evaluation instrument for the total customer experience. Behaviour \& Information Technology, Vol 25, No 2, March-April 2006, pp 189 - 203.

[17] Picard, R.W. Affective Computing for HCI. Ergonomics and User Interfaces 1999.

[18] Preece, J; Rogers, Y; and Sharp, H. Interaction Design: Beyond Human-Computer Interaction. Wiley, USA, 2002.

[19] Simon, V.M. Emotional Participation in DecisionMaking. Psychology in Spain, Vol 2, No. 1, pp 100-107.

[20] Swallow, D; Blythe, M and Wright, P. Grounding Experience: Relating Theory and Method to Evaluate the User Experience of Smartphones. Proceedings of the 2005 Annual Conference on European Association of Cognitive Ergonomics, Chania, Greece. Session: On theories, methods and techniques. Vol. 132, 2005, pp 1- 98.

[21] Toms, E.G; Dufour, C and Hesemeier, S. Measuring the User's Experience with Digital Libraries. JCDL '04, June 7-11, 2004, Tucson, Arizona, USA.

[22] Wiberg, C. Usability and Fun: An overview of relevant research in the HCI community. http://www.sics.se/ kia/evaluating_affective_interfaces_/ Wiberg_2.doc. 